Editorial

\title{
Biological and Clinical Significance of Androgens and Androgen Receptor in Prostate Cancer
}

\author{
Shahriar Koochekpour $\bowtie$ \\ Departments of Cancer Genetics and Urology, Center for Genetics and Pharmacology, Roswell Park Cancer Institute, Elm and Carlton \\ Streets, Buffalo, NY 14263, USA.
}

\begin{abstract}
$\square$ Corresponding author: Departments of Cancer Genetics and Urology, Center for Genetics and Pharmacology, Roswell Park Cancer Institute, Elm and Carlton Streets, Buffalo, New York, United States of America. Tel: 716-845-3345; Fax: 716-845-1698; Email: Shahriar.Koochekpour@Roswellpark.org
\end{abstract}

(C) Ivyspring International Publisher. This is an open-access article distributed under the terms of the Creative Commons License (http://creativecommons.org/ licenses/by-nc-nd/3.0/). Reproduction is permitted for personal, noncommercial use, provided that the article is in whole, unmodified, and properly cited.

Received: 2014.05.05; Accepted: 2014.05.09; Published: 2014.06.10

This special issue provides an update on different biological and clinical aspects of adrenal androgens, androgen biosynthetic pathways, AR-signaling, inter-racial differences in AR mutations, and clinical success and failure of antiandrogens and anti-AR therapies in prostate cancer (PCa). Androgen receptor (AR), a nuclear steroid receptor and transcription factor, has a central role in the normal growth and development of the prostate gland, in prostate carcinogenesis, and in the castrate-resistant (CR) progression of PCa [1]. Understanding the critical molecular events that lead PCa cells to resist antiandrogens or anti-AR therapies is essential in developing successful treatments for CR or advanced stages of the disease $[2,3]$. In a relatively significant number of PCa patients, the AR is overexpressed, mutated or genomically amplified [4]. In addition, the failure of androgen-deprivation therapy (ADT) and recurrence of PCa with lethal phenotypes highlighted the importance of intratumoral (intracrine) androgen biosynthesis and metabolic pathways.

Dr. Heemers [5] in her review describes current ADT that acts at the pre-receptor and the receptor levels and presents the potential of interference with the molecular recognition and regulation of AR-dependent transcription and the action of AR target genes at the post-receptor level. The feasibility of developing post-receptor level ADT has been demonstrated by additional experimental evidence. Dr. Heemers explores the potential limitations to clinical application of post-receptor level ADT and additional avenues for the development of novel and more PCa-specific or selective forms of ADT.
Growing evidence indicates that microtubule-targeting chemotherapy (such as docetaxel and cabazitaxel) and androgen/AR signaling targeting agents (abiraterone and enzalutamide) can affect the cellular localization and transcriptional activity of AR. This provides a molecular basis for the cross-resistance that can emerge between taxanes and antiandrogens and between the antiandrogens, abiraterone and enzalutamide, as well as the therapeutic responses driven by the treatment sequence for CR-PCa. In the manuscript by Kyprianou et al [6], they review the current knowledge of the mechanisms by which (a) the AR signaling via regulation of epithelial-mesnchymal transition drives therapeutic resistance in CR-PCa and metastatic disease and (b) the therapeutic resistance to andtiandrogens and /or taxane-based chemotherapy can be overcome in metastatic CR-PCa.

Drs. Mohler and Fiandalo's review [7] describes the evolution of the adrenal androgen blockade, how new androgen measurement methods have advanced our understanding of androgen metabolism, and how further understanding of the backdoor pathway of androgen biosynthesis pathway may lead to interventions that might extend survival for CR-PCa even more.

Emerging data demonstrate that the novel 5a-reduced derivative of adrenal steroids can activate the wild-type AR, and suggest that a larger pool of residual androgens may be available to activate the $A R$ in CR-PCa than was previously realized. Importantly, the significant role of $11 \mathrm{OH}-5 \mathrm{a}-\mathrm{DHT}$ and $11 \mathrm{~K}-5 \mathrm{a}-\mathrm{DHT}$ in activating the wild-type AR suggests 
that inhibiting the enzymes unique to the production of these metabolites may significantly decrease the overall androgenicity of the CR-PCa tumor microenvironment and thereby improve clinical outcomes. Dr. Mostaghel [8] provides a detailed review on contemporary pathways of androgen biosynthesis covering adrenal androgen synthesis, classical, backdoor, and 5a-dione pathway of androgen metabolism.

Dr. Zhao et al. describe the regulatory effects of the A- and O-classes of the forkhead box (FOX) proteins on AR-transcriptional activity, androgen-dependent or -stimulated PCa growth, and their contribution to taxane-mediated inhibition of AR and CR-PCa growth [9]. While increased FOXA1 expression leads to an overactive AR complex that eventually hypersensitizes CR-PCa cells to very low levels of androgens, the FOXO1 protein blocks AR activity by direct and indirect mechanisms. Their review focuses on recent advances in the epigenetic, mechanistic and clinically relevant aspects of regulation of the AR by FOXA1 and FOXO1 factors in PCa.

A fundamental question in the field has been how the transcriptional activity of AR differs in the CR- vs. androgen-dependent setting. The manuscript by Dr. Gelman [10] addresses a growing body of literature showing that Src-family (SFK) and Ack1 tyrosine kinases and the serine/threonine kinase, PKC, can directly phosphorylate AR, thereby activating transcriptional activity in $\mathrm{CR}$ conditions. These kinases are likely activated by upstream receptor tyrosine kinases, such as EGFR and IGFR, which themselves are upregulated and/or activated during the progression to CR-PCa. These findings have formed the rationale for pre-clinical and clinical studies to combine SFK/Ack1/PKC antagonists with drugs targeting the androgen/AR axis in the treatment or prevention of CR-PCa.

In the article by Dr. Vander Griend et al [11], the authors document that in the normal human prostate, AR functions to block the proliferation of prostate epithelial cells and drive their differentiation into secretory-luminal cells. Moreover, their study provides mechanistic data demonstrating that such terminal growth inhibition does not require RB, p21, or p27, occurs via an S-phase kinase-associated protein 2 (SKP-2) independent mechanism, requires AR-DNA binding and can be overcome by c-Myc overexpression. These data support a model whereby the normal function of AR is as a cell context-dependent growth suppressor of prostate epithelial cells and that this function is characteristically subverted by malignant prostate cells where AR now functions as an oncogene.

Very limited knowledge is available for genetic alterations in AR in African American (AA) men who present with a higher PCa incidence and mortality rate than Caucasian Americans (CAs). Koochekpour et al report a higher rate for somatic AR missense mutations in primary tumors or germline mutations and polymorphisms in AAs than in CAs [12]. They discovered that transient silencing of the previously discovered AR599 (Ser>Gly) mutation did not affect cell proliferation but significantly decreased migratory and invasive phenotypes of E006AA, a primary AA-PCa cell line. These data provide experimental evidence for the coexisting "loss-of-function" (cell proliferation) and "gain-of-function" (migration and invasion) phenotypes secondary to a missense point mutation and highlights the complexity of predicting functional outcome of site-specific changes of AR gene in multifocal malignant prostate tissues.

We hope that this special issue edition sheds some light on the complex biology of AR, androgen biosynthetic pathways and important signaling mechanisms regulating AR activity in PCa.

\section{Competing Interests}

The author has declared that no competing interest exists.

\section{References}

1. Mitsiades N. A road map to comprehensive androgen receptor axis targeting for castration-resistant prostate cancer.. Cancer Res 2013; 73: 4599-605. Review.

2. Hieronymus H, Sawyers CL. Traversing the genomic landscape of prostate cancer from diagnosis to death. Nat Genet 2012; 44: 613-4.

3. Heidegger I, Massoner P, Eder IE, et al. Novel therapeutic approaches for the treatment of castration-resistant prostate cancer. J Steroid Biochem Mol Biol 2013; 138: 248-56. Review.

4. Barbieri CE, Bangma CH, Bjartell A, et al. The mutational landscape of prostate cancer. Eur Urol 2013; 64: 567-76.

5. Heemers HV. Targeting Androgen Receptor Action for Prostate Cancer Treatment: Does the Post-Receptor Level Provide Novel Opportunities? Int J Biol Sci 2014; 10(6):576-587. doi:10.7150/ijbs.8479.

6. Kahn B, Collazo J, Kyprianou N. Androgen Receptor as a Driver of Therapeutic Resistance in Advanced Prostate Cancer. Int J Biol Sci 2014; 10(6):588-595. doi:10.7150/ijbs.8671.

7. Fiandalo MV, Wilton J, Mohler JL. Roles for the Backdoor Pathway of Androgen Metabolism in Prostate Cancer Response to Castration and Drug Treatment. Int J Biol Sci 2014; 10(6):596-601. doi:10.7150/ijbs.8780.

8. Mostaghel EA. Beyond T and DHT - Novel Steroid Derivatives Capable of Wild Type Androgen Receptor Activation. Int J Biol Sci 2014; 10(6):602-613. doi:10.7150/ijbs.8844.

9. Zhao Y, Tindall DJ, Huang H. Modulation of Androgen Receptor by FOXA1 and FOXO1 Factors in Prostate Cancer. Int J Biol Sci 2014; 10(6):614-619. doi:10.7150/ijbs.8389.

10. Gelman IH. Androgen Receptor Activation in Castration-Recurrent Prostate Cancer: The Role of Src-Family and Ack1 Tyrosine Kinases. Int J Biol Sci 2014; 10(6):620-626. doi:10.7150/ijbs.8264.

11. Griend DJV, Litvinov IV, Isaacs JT. Conversion of Androgen Receptor Signaling From a Growth Suppressor in Normal Prostate Epithelial Cells to an Oncogene in Prostate Cancer Cells Involves a Gain of Function in c-Myc Regulation. Int J Biol Sci 2014; 10(6):627-642. doi:10.7150/ijbs.8756.

12. Koochekpour S, Buckles E, Shourideh M, Hu S, Chandra D, Zabaleta J, Attwood K. Androgen Receptor Mutations and Polymorphisms in African American Prostate Cancer. Int J Biol Sci 2014; 10(6):643-651. doi:10.7150/ijbs.8974. 